\title{
Finite Dimensional Representations of the Quantum Analog of the Enveloping Algebra of a Complex Simple Lie Algebra
}

Marc Rosso

Centre de Mathématiques, Ecole Polytechnique, F-91 128, Palaiseau Cédex, France

\begin{abstract}
Let $\mathscr{G}$ be a complex simple Lie algebra. We show that when $t$ is not a root of 1 all finite dimensional representations of the quantum analog $U_{t} \mathscr{G}$ are completely reducible, and we classify the irreducible ones in terms of highest weights. In particular, they can be seen as deformations of the representations of the (classical) $U \mathscr{G}$.
\end{abstract}

\section{Introduction}

To each complex simple Lie algebra $\mathscr{G}$, Jimbo associates the quantum analog of its enveloping algebra, let $U_{t} \mathscr{G}$, where $t$ is a non-zero parameter, as follows (see also Drinfeld $[2,3])$ :

Let $\left(a_{i j}\right)_{1 \leqq i, j \leqq N}$ be the Cartan matrix of $\mathscr{G}$ and $\left(\alpha_{i}\right)_{1 \leqq l \leqq N}$ a basis of simple roots; $U_{t} \mathscr{G}$ is the C-algebra generated by $\left(k_{i}^{ \pm 1}, e_{i}, f_{i}\right)_{1 \leqq i \leqq N}$ with relations:

$$
\begin{gathered}
k_{i} \cdot k_{i}^{-1}=k_{i}^{-1} \cdot k_{i}=1 ; \quad k_{i} k_{j}=k_{j} k_{i}, \\
k_{i} e_{j} k_{i}^{-1}=t_{i}^{a_{1 j}} e_{j} ; \quad k_{i} f_{j} k_{i}^{-1}=t_{i}^{-a_{\imath \jmath}} f_{j}, \\
{\left[e_{i}, f_{j}\right]=\delta_{i j} \frac{k_{i}^{2}-k_{i}^{-2}}{t_{i}^{2}-t_{i}^{-2}},} \\
\sum_{v=0}^{1-a_{i j}}(-1)^{v}\left[\begin{array}{c}
1-a_{i j} \\
v
\end{array}\right]_{t_{i}^{2}} e_{i}^{1-a_{i j}-v} e_{j} e_{i}^{v}=0 \quad \text { for } i \neq j, \\
\sum_{v=0}^{1-a_{i j}}(-1)^{v}\left[\begin{array}{c}
1-a_{i j} \\
v
\end{array}\right]_{t_{i}^{2}} f_{i}^{1-a_{l j}-v} f_{j} f_{i}^{v}=0 \quad \text { for } i \neq j,
\end{gathered}
$$

where $t_{i}=t^{\left(\alpha_{i} \mid \alpha_{i}\right) / 2},(\mid)$ being the invariant inner product on $\oplus \mathbf{C} \alpha_{i}$, with $\left(\alpha_{i} \mid \alpha_{i}\right) \in \mathbf{Z}$.

$$
\left[\begin{array}{c}
m \\
n
\end{array}\right]_{t}= \begin{cases}\frac{\left(t^{m}-t^{-m}\right)\left(t^{m-1}-t^{-(m-1)}\right) \cdots\left(t^{m-n+1}-t^{-(m-n+1)}\right)}{\left(t-t^{-1}\right)\left(t^{2}-t^{-2}\right) \cdots\left(t^{n}-t^{-n}\right)} & \text { for } m>n>0, \\
1 \quad \text { for } n=0 \text { or } m=n . & \end{cases}
$$


So $t_{i}^{a_{i j}}=t_{j}^{a j i}=t^{\left(\alpha_{i} \mid \alpha_{j}\right)}$. There is a coproduct: $\Delta: U_{t} \mathscr{G} \rightarrow U_{t} \mathscr{G} \otimes U_{t} \mathscr{G}$ defined by:

$$
\begin{gathered}
\Delta\left(k_{i}^{ \pm 1}\right)=k_{i}^{ \pm 1} \otimes k_{i}^{ \pm 1}, \\
\Delta\left(e_{i}\right)=e_{i} \otimes k_{i}^{-1}+k_{i} \otimes e_{i} ; \quad \Delta\left(f_{i}\right)=f_{i} \otimes k_{i}^{-1}+k_{i} \otimes f_{i},
\end{gathered}
$$

and $U_{t} \mathscr{G}$ is a Hopf algebra with antipode $S$ and augmentation $\varepsilon$ respectively defined by:

$$
\begin{gathered}
S\left(k_{i}\right)=k_{i}^{-1}, \quad S\left(e_{i}\right)=-t_{i}^{-2} e_{i}, \quad S\left(f_{i}\right)=-t_{i}^{2} f_{i}, \\
1=\varepsilon\left(k_{i}\right)=\varepsilon\left(k_{i}^{-1}\right) ; \quad \varepsilon\left(e_{i}\right)=\varepsilon\left(f_{i}\right)=0 .
\end{gathered}
$$

From now on, we shall assume that $t$ is not a root of 1 and we shall study the finite dimensional representations of $U_{t} \mathscr{G}$.

In [4], Jimbo has shown that, for $\mathscr{G}=\operatorname{sl}(N+1)$, any irreducible finite dimensional representation can be deformed in an irreducible representation of $U_{t} \mathscr{G}$. We shall show, using analogs of highest weight modules, that all finite dimensional representations are essentially obtained in this way (after possibly tensoring by a 1-dimensional representation) and that all finite dimensional representations are completely reducible.

The paper is organised as follows: in sect. II, we give some lemmas on the general structure of $U_{t} \mathscr{G}$, in particular showing a triangular decomposition: $U_{t} \mathscr{G}=U_{t} n_{-} \otimes \mathbf{C}[T] \otimes U_{t} n_{+}$as vector spaces (see notations below). In sect. III, we give general remarks on finite dimensional representations of $U_{t} \mathscr{G}$, which lead us to highest weights. In Sect. IV we treat the case of $U_{t} \operatorname{sl}(2)$, which is used in sect. $\mathrm{V}$ to get the result for any $U_{t} \mathscr{G}$.

\section{Notations}

- $T$ is the subgroup of the group of invertible elements of $U_{t} \mathscr{G}$, generated by the $k_{i}$ 's, and $\mathbf{C}[T]$ is its group algebra.

- $U_{t} n_{+}$(respectively $U_{t} n_{-}$) is the subalgebra of $U_{t}^{\mathscr{G}}$ generated by the $e_{i}$ 's (respectively by the $f_{i}$ 's).

- $U_{t} b_{+}$(respectively $U_{t} b_{-}$) is the subalgebra of $U_{t} \mathscr{G}$ generated by the $e_{i}$ 's and $k_{i}^{ \pm 1}$ 's). (respectively by $e_{i}$ 's and $k_{i}^{ \pm 1}$ 's).

$$
\cdot A=\bigoplus_{i=1}^{N} \mathbf{Z} \alpha_{i} \text { is the root lattice, and } Q_{+}=\bigoplus_{i=1}^{N} \mathbf{N} \alpha_{i} \text {. }
$$

\section{About the Structure of $U_{t}^{\mathscr{G}}$}

\section{Q-Gradation}

Proposition 1. The action of $k_{i}$ 's by conjugation gives a $Q$-gradation on $U_{t} \mathscr{G}, U_{t} b_{ \pm}$, $U_{t} n_{ \pm}$as follows: a monomial $\xi$ in the generators $e_{i}, f_{i}, k_{i}$, is said to be the degree $\alpha=\sum_{1}^{N} n_{i} \alpha_{i}, n_{i} \in \mathbf{Z}$ iff:

$$
\forall i=1, \ldots, N \quad k_{i} \xi k_{i}^{-1}=t_{i}^{\left(x_{2} \mid \alpha\right)} \xi .
$$

Proof. Let us note first that the $t_{i}^{\left(\alpha_{l} \mid \alpha\right)}, 1 \leqq i \leqq N$, completely determine $\alpha$ : as 
$\left(\alpha_{i} \mid \alpha\right) \in \mathbf{Z}$ and $t$ is not a root of 1 , the $t_{i}^{\left(\alpha_{i} \mid \alpha\right)}$ determine the integers $\left(\alpha_{i} \mid \alpha\right)$ which in turn determine $\alpha$ as $(\mid)$ is non-degenerate.

As each polynomial $\xi$ where $e_{i}$ appears $n_{i}$ times and $f_{i} m_{i}$ times is clearly of degree $\alpha=\sum_{1}^{N}\left(n_{i}-m_{i}\right) \alpha_{i}$, we see that $U_{t} \mathscr{G}, U_{t} b_{ \pm}, U_{t} n_{ \pm}$are sums of their subspaces of degree.

Remark. $U_{t} \mathscr{G} \otimes U_{t} \mathscr{G}$ is then $Q \times Q$-graded, and also $Q$-graded via the total gradation.

$\Delta: U_{t} \mathscr{G} \rightarrow U_{t} \mathscr{G} \otimes U_{t} \mathscr{G}$ is a morphism of $Q$-graded algebras.

Lemma 1. $\forall\left(m_{1}, \ldots, m_{n}\right) \in \mathbf{N}^{N}, e_{1}^{m_{1}} \cdots e_{N}^{m_{N}}$ is non-zero in $U_{t} \mathscr{G}$.

Proof.

a) There is always the fundamental representation of $U_{t} \mathscr{G}$ (given by the same formulas as the fundamental representation of $\mathscr{G}$, see Jimbo [6]) in which the $e_{i}$ 's are non-zero. (One can also mimic the proof in Humphreys [4] p. 97-99).

b) $\forall i \in\{1, \ldots, N\}, \forall m \in \mathbf{N} e_{i}^{m} \neq 0$.

As $\Delta$, and also the $\Delta^{(m)}=\left(\Delta \otimes \mathrm{Id}^{\otimes(m-1)} \circ\left(\Delta \otimes \operatorname{Id}^{\otimes(m-2)} \circ \ldots \circ \Delta\right.\right.$, are injective, it is enough to show that $\Delta^{(m)}\left(e_{i}^{m}\right) \neq 0$. Using the $Q^{m}$-gradation of $\left(U_{t} \mathscr{G}\right)^{\otimes m}$, it is enough to check that the component of degree $\left(\alpha_{i}, \ldots, \alpha_{i}\right)$ is non-zero.

Now, $\Delta^{(m)}\left(e_{i}\right)=u_{1}+\cdots+u_{m}$, where $u_{r}=k_{i} \otimes \cdots \otimes k_{i} \otimes e_{i} \otimes k_{i}^{-1} \otimes \cdots \otimes k_{i}^{-1}\left(e_{i}\right.$ at the r-th position) and $u_{s} u_{r}=t_{i}^{4} u_{r} u_{s}$ for $r<s$. So, one computes $\Delta^{(m)}\left(e_{i}^{(m)}\right)=$ $\left[\Delta^{(m)}\left(e_{i}\right)\right]^{m}$ by the $t_{i}^{4}$-multinomial formula:

$$
\left[\Delta^{(m)}\left(e_{i}\right)\right]^{m}=\sum_{n_{1}+\cdots+n_{m}=m} \frac{\phi_{m}\left(t_{i}^{4}\right)}{\phi_{n_{1}}\left(t_{i}^{4}\right) \cdots \phi_{n_{m}}\left(t_{i}^{4}\right)} u_{1}^{n_{1}} \cdots u_{m}^{n_{m}},
$$

and one gets the term of degree $\left(\alpha_{i}, \ldots, \alpha_{i}\right)$ for $n_{1}=\cdot=n_{m}=1$. So, it is

$$
\frac{\phi_{m}\left(t_{i}^{4}\right)}{\left[\phi\left(t_{i}^{4}\right)\right]^{m}} u_{1} \cdots u_{m}=\frac{\left(t_{i}^{2}-t_{i}^{-2}\right)\left(t_{i}^{4}-t_{i}^{-4}\right) \cdots\left(t_{i}^{2 m}-t_{i}^{-2 m}\right)}{\left(t_{i}^{2}-t_{i}^{-2}\right)^{m}}
$$

Now, as $k_{i}$ is invertible, we see that, $e_{i} k_{i}^{m-1} \otimes \cdots \otimes e_{i} k_{i}^{-(m-1)}$ is nonzero.

c) Let $\left(m_{1}, \ldots, m_{N}\right) \in \mathbf{N}$. In order to see that $e_{1}^{m_{1}} \cdots e_{N}^{m_{n}} \neq 0$, it is enough to consider the component of degree $\left(m_{1} \alpha_{1}, \ldots, m_{N} \alpha_{N}\right)$ of $\Delta^{(N)}\left(e_{1}^{m_{1}} \cdots e_{N}^{m_{N}}\right)$. But it is:

$$
e_{1}^{m_{1}} k_{2}^{m_{2}} \cdots k_{N}^{m_{N}} \otimes k_{1}^{-m_{1}} e_{2}^{m_{2}} \cdots k_{N}^{m_{N}} \otimes \cdots \otimes k_{1}^{-m_{1}} \cdots k_{N-1}^{-m_{N-1}} e_{N}^{m_{N}}
$$

which is non-zero according to $b$ ).

\section{A Basis for $\mathbf{C}[T]$}

For $\alpha=\sum_{1}^{N} n_{i} \alpha_{i} \in Q$, let $k_{\alpha}=k_{1}^{n_{1}} \cdots k_{N}^{n_{N}}$.

Lemma 2. The $k_{\alpha}$ 's, $\alpha \in Q$, are linearly independent.

Proof. Suppose $\sum_{\text {finite }} \lambda_{\alpha} k_{\alpha}=0, \lambda_{\alpha} \in \mathbf{C}^{*}$. As one can always multiply by a $k_{\beta}$ (with a suitable $\beta$ ), one can assume that the $\alpha$ 's in the finite sum belong to $Q_{+}$. 


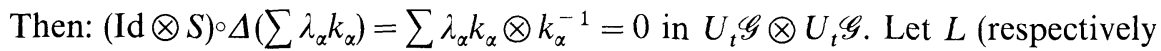
$R)$ be the left (respectively right) regular representation of $U_{t} \mathscr{G}$.

So: $\sum \lambda_{\alpha} L\left(k_{\alpha}\right) \circ R\left(k_{\alpha}^{-1}\right)=0$ in $\operatorname{End}\left(U_{t} \mathscr{G}\right)$.

Evaluating on $e_{1}^{m_{1}} \ldots e_{N}^{m_{N}},\left(m_{1}, \ldots, m_{N}\right) \in \mathbf{N}^{N}$, one gets:

$$
\sum \lambda_{\alpha} t^{\left.(\alpha) \sum m_{1} \alpha_{1}\right)}=0 \quad \forall\left(m_{1}, \ldots, m_{N}\right) \in \mathbf{N}^{N} .
$$

As we can also evaluate on $e_{1}^{k m_{1}} \cdots e_{N}^{k m_{N}}$ for each $k \in \mathbf{N}$, we see that $t$ and all its power $t^{k}$ are roots of a certain Laurent polynomial. As $t$ is not a root of 1 , its powers are 2 by 2 distincts so the Laurent polynomial must be 0 . So

$$
\sum_{\alpha,\left(\alpha \mid \sum m_{1} \alpha_{i}\right)=\text { fixedvalue }} \lambda_{\alpha}=0 .
$$

Using this remark, we shall give a proof by induction on the number $p$ of terms in the sum (recall we have assumed $\lambda_{\alpha} \in \mathbf{C}^{*}$ )

- the case $p=1$ is clear

- let us suppose the result true for $p$ terms ( $p \geqq 1$ ), and suppose there are $p+1$ terms: $\alpha^{(0)}, \ldots, \alpha^{(p)}$.

It is enough to show that there exists $\left(m_{1}, \ldots, m_{N}\right) \in \mathbf{N}^{N}$ such that:

$$
\left(\alpha^{(o)} \mid \sum m_{i} \alpha_{i}\right) \notin\left\{\left(\alpha^{(k)} \mid \sum m_{i} \alpha_{i}\right) k=1, \ldots, p\right\}
$$

(because then the argument on Laurent polynomials gives $\lambda_{\alpha(0)}=0$, and we are back to a sum with $p$ terms).

$\left(^{*}\right)$ reads: $\exists\left(m_{1}, m_{N}\right) \in \mathbf{N}^{N}$ such that: $\forall k=1, \ldots, p\left(\alpha^{(o)}-\alpha^{(k)}, \sum_{1}^{N} m_{i} \alpha_{i}\right) \neq 0$. But the $\left(\alpha^{(o)}-\alpha^{(k)}, \cdot\right)$ are non-zero linear forms on $h^{*}$, which determine $p$ hyperplanes in $h^{*}$. We have to see that there is a point of $Q_{+}$outside the union of these hyperplanes. The proof is exactly the same as the classical one showing that any vector space on a field of characteristic 0 cannot be the union of a finite number of hyperplanes.

4. Basis for $U_{t} n_{ \pm}$

As the vector space $U_{t} n_{+}$is generated by monomial in the $e_{i}$ 's, there is a basis of $U_{t} n_{+}$whose elements are some of these monomials; one can also assume that the monomials in this basis having a given $Q$-degree form a basis of the corresponding $Q$-component of $U_{t} n_{+}$.

Let $\left(E_{r}\right)_{r \in I}$ this basis.

Lemma 3. $\left(E_{t} \cdot k_{\alpha}\right)_{r \in I, \alpha \in Q}$ is a basis of $U_{t} b_{+}$. So $U_{t} b_{+} \simeq U_{t} n_{+} \otimes \mathbf{C}[T]$ as vector spaces.

Proof. According to the defining relations of $U_{t} \mathscr{G}$, these elements generate $U_{t} b_{+}$. Let us show they are linearly independent.

Suppose $\sum \lambda_{r} E_{r} k_{\alpha_{r}}=0, \lambda_{r} \in \mathbf{C}^{*}$. One can assume that all the terms have the same $Q$-degree $\beta$. The term of degree $(\beta, 0)$ in $\Delta\left(\sum \lambda_{r} E_{r} k_{\alpha_{r}}\right)$ must be 0 , so:

$$
\begin{aligned}
\sum \lambda_{r} E_{r} k_{r} \otimes k_{\beta} k_{\alpha_{r}} & =0, \\
\sum_{\alpha}\left(\sum_{\left\{r \mid \alpha_{r}=\alpha\right\}} \lambda_{r} E_{r} k_{\alpha_{r}}\right) \otimes k_{\alpha} k_{\beta} & =0 .
\end{aligned}
$$


As $k_{\alpha}$ 's, for distinct $\alpha$ 's, are independent:

$$
\sum_{\left\{r / \alpha_{r}=\alpha\right\}} \lambda_{r} E_{r} k_{\alpha}=0, \text { so } \sum \lambda_{r} E_{r}=0 \text { and } \forall r \lambda_{r}=0 .
$$

Remark. Let $\theta$ the algebra automorphism given by $\theta\left(e_{i}\right)=-f_{i}, \theta\left(f_{i}\right)=-e_{i}$, $\theta\left(k_{i}\right)=k_{i}^{-1}$.

Let $F_{r}=\theta\left(E_{r}\right)$. Then $\left(F_{r}\right)_{r \in I}$ is a basis of $U_{t} n_{-}$having the same properties as $\left(E_{r}\right)_{r \in I}$.

\section{The Triangular Decomposition of $U_{t} \mathscr{G}$}

Proposition 2. $\left(E_{r} \cdot F_{r^{\prime}} \cdot k_{\alpha}\right)_{\left(r, r^{\prime}, \alpha\right) \in I \times I \times Q}$ is a basis of $U_{t} \mathscr{G}$. So $U_{t} \mathscr{G} \simeq U_{t} n_{-} \otimes \mathbf{C}[T] \otimes U_{t} n_{+}$ as vector spaces and $U_{t} \mathscr{G}$ is a free $U_{t} b_{+}$-module.

Proof. It is enough to show the linear independence. Suppose $\sum \lambda_{r, r^{\prime}, \alpha} E_{r} . F_{r^{\prime}} k_{\alpha}=0$, $\lambda_{r, r^{\prime}, \alpha} \in \mathbf{C}^{*}$. For $r \in I$, let $\alpha_{r}$ the $Q$-degree of $E_{r}$ (and- $\alpha_{r^{\prime}}$ for $F_{r^{\prime}}$ ). Then, the $Q$-degree of $E_{r} \cdot F_{r^{\prime}} \cdot k_{\alpha}$ is $\alpha_{r}-\alpha_{r^{\prime}}$ and we can assume that the couples $\left(r, r^{\prime}\right)$ in the sum are such that $\alpha_{r}-\alpha_{r^{\prime}}=$ constant.

We shall use an order relation $\leqq$ on $Q$, defined as follows:

for $\alpha=\sum n_{i} \alpha_{i} \in Q$, let $m_{i}(\alpha)=n_{i}, l(\alpha)=\sum_{1}^{N} m_{i}(\alpha) \in \mathbf{Z}$. For $\alpha \neq \alpha^{\prime}$, we say that $\alpha<\alpha^{\prime}$ if:

a) $l(\alpha)<l\left(\alpha^{\prime}\right)$ or

b) $l(\alpha)=l\left(\alpha^{\prime}\right)$ and the smallest index $i$ such that $m_{i}(\alpha) \neq m_{i}\left(\alpha^{\prime}\right)$ verifies: $m_{i}(\alpha)<$ $m_{i}\left(\alpha^{\prime}\right)$. This order is total, and compatible with the addition.

Now, consider $I_{0}=\left\{r \in I /\right.$ the degree $\alpha_{r}$ of $E_{r}$ is maximal for $\left.\leqq\right\}$. Then, in $\Delta\left(\sum \lambda_{r, r^{\prime}, \alpha} E_{r} F_{r^{\prime}} k_{\alpha}\right)=0$, the component of $Q \times Q$-degree (maximal, minimal) must be 0 :

$$
\sum_{r \in I_{0}} \lambda_{r, r^{\prime}, \alpha}\left(E_{r} k_{\alpha_{r^{\prime}}} \otimes k_{\alpha_{r}}^{-1} F_{r^{\prime}}\right) k_{\alpha} \otimes k_{\alpha}=0 .
$$

Here $\alpha_{r}$ is fixed, so $\alpha_{r^{\prime}}$ also,

$$
\begin{gathered}
\sum_{r \in I_{0}} \lambda_{r, r^{\prime}, \alpha}\left(E_{r} k_{\alpha} \otimes F_{r^{\prime}} k_{\alpha}\right)=0 \\
\sum_{\left(r^{\prime}, \alpha\right) 2 \text { by } 2 \text { distinct }}\left(\sum_{r \in I_{0},\left(\boldsymbol{r}^{\prime}, \alpha\right) \text { fixed }} \lambda_{r, r^{\prime}, \alpha} E_{r} k_{\alpha}\right) \otimes F_{r^{\prime}} k_{\alpha}=0 .
\end{gathered}
$$

As the $F_{r^{\prime}} k_{\alpha}$ are independent, $\forall\left(r^{\prime}, \alpha\right)$ fixed $\sum_{r \in I_{0}} \lambda_{r, r^{\prime}, \alpha} E_{r} k_{\alpha}=0$, so $\lambda_{r, r^{\prime}, \alpha}=0$.

\section{General Remarks on the Finite Dimensional Representations}

Let $\rho$ a representation of $U_{t} \mathscr{G}$ in the finite dimensional vector space $V$.

Lemma 4. 1. The operators $\rho\left(e_{i}\right), \rho\left(f_{i}\right)(1 \leqq i \leqq N)$ are nilpotent.

2. If $\rho$ is irreducible, the $\rho\left(k_{i}\right)$ 's are simultaneously diagonalisable and $V=\oplus V_{\mu}$, where, for $\mu=\left(\mu_{1}, \ldots, \mu_{N}\right)$,

$$
V_{\mu}=\left\{v \in V / \forall i \quad \rho\left(k_{i}\right) v=\mu_{i} v .\right\}
$$

Remark. Such a $\mu$ defines a character $\mu: T \rightarrow \mathbb{C}^{*}$, this allows us to speak about weights of the representation. 
Proof. 1. For $1 \leqq i \leqq N$, the relation $\rho\left(k_{i}\right) \rho\left(e_{i}\right) \rho\left(k_{i}\right)^{-1}=t^{\left(\alpha_{i} \mid \alpha_{i}\right)} \rho(e)$ shows that if the spectrum of $\rho\left(e_{i}\right)$ contains a non-zero element, it contains an infinity of elements. So, this spectrum is $\{0\}$ and $\rho\left(e_{i}\right)$ is nilpotent. Same proof for $\rho\left(f_{i}\right)$.

2. As the $\rho\left(k_{i}\right)$ commute, they have a common eigenvector $v$ and we have to see that each is diagonalisable. Let $E=\left\{W\right.$ subspace of $V, \operatorname{dim} W \geqslant 1 / \forall i,\left.\rho\left(k_{i}\right)\right|_{W}$ diagonalisable $E \neq \varnothing$ as $\mathbb{C} \cdot v \in E$. Let $W \in E$ of maximal dimension and suppose $\operatorname{dim} W<\operatorname{dim} V$ :

a) if $W$ is invariant under $\rho\left(e_{i}\right)$ and $\rho\left(f_{i}\right)$, we must have $W=V$ due to the irreducibility of $V$.

b) assume there exists $w \in W$ and $j \in\{1, \ldots, N\}$ such that $\rho\left(e_{j}\right) w \notin W$. (The case $\rho\left(f_{j}\right) w \notin W$ is similar.) As $W=\oplus W_{\mu}$, where $W_{\mu}=\left\{w / \rho\left(k_{i}\right) w=\mu_{i} w\right\}$, we can assume that $w \in W_{\mu}$ for a certain $\mu$. Then $\rho\left(k_{i}\right) \rho\left(e_{j}\right) w=t_{i}^{a_{i j}} \rho\left(e_{j}\right) \rho\left(k_{i}\right) w=\mu_{i} t_{i}^{a_{i_{j}}} \rho\left(e_{j}\right) w$. So $w^{\prime}=$ $\rho\left(e_{j}\right) w$ is a common eigenvector of all $\rho\left(k_{i}\right)$ 's and $W^{\prime}=W \oplus \mathbb{C} w^{\prime}$ belongs to $E$, with $\operatorname{dim} W^{\prime}>\operatorname{dim} W$. Contradiction.

Definition. A vector $v \in V \backslash\{0\}$ is said a highest weight vector if there exists $\lambda=\left(\lambda_{1}, \ldots, \lambda_{N}\right) \in\left(\mathbf{C}^{*}\right)^{N}$ such that: $\rho\left(k_{i}\right) v=\lambda_{i} v \forall i=1, \ldots, N$,

$$
\rho\left(e_{i}\right) v=0 \forall i=1, \ldots, N .
$$

Proposition 3. For each finite dimensional representation $(\rho, V)$, there is at least a highest weight vector in $V$.

Proof. a) As the $\rho\left(k_{i}\right)$ 's are simultaneously trigonalisable, the set of weights $P$ is non-empty; The subvectorspace $V^{\prime} \approx \oplus V_{\mu}$ of $V$ is non-zero and invariant under $U_{t} \mathscr{G}$. We consider the subrepresentation of $U_{t} \mathscr{G}$ in $V^{\prime}$ and look for a highest weight vector in $V^{\prime}$.

b) In $V^{\prime}$, we only have to show that $V_{0}=\bigcap_{1}^{N} \operatorname{Ker} \rho\left(e_{j}\right)$ is not zero (as it is invariant under the $\rho\left(k_{i}\right)$ 's, they have a common eigenvector in it). This follows classically from the lemma:

Lemma 5. There exists an integer $M$ such that: $\forall j_{1}, \ldots, j_{p} \in\{1, \ldots, N\}, \rho\left(e_{j_{1}}\right) \cdots \rho\left(e_{j_{p}}\right)=$ 0 in End $V^{\prime}$ as soon as $p \geqq M$.

Proof. It is enough to check that: $\forall \mu \in P, \forall v \in V_{\mu}^{\prime}, \rho\left(e_{j_{1}}\right) \cdots \rho\left(e_{j_{p}}\right) v=0$ for $p$ big enough.

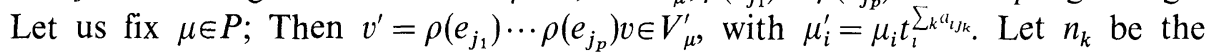
number of times $e_{k}$ appears in $\left\{e_{j_{1}}, \ldots, e_{j_{p}}\right\} ; \mu_{i}^{\prime}=\mu_{i} t_{i}^{\sum_{n_{k}} a_{i k}}$. As $V^{\prime}$ is finite dimensional, there is only a finite number of weights $\mu, \mu^{(1)}, \ldots, \mu^{(2)}$, and it is enough to see that for $p \geqslant M, \mu^{\prime}$ is not in this list for $i \in\{1, \ldots, N\}$, let $x_{i}^{(s)}=\mu_{i}^{(s)} / \mu_{i}$; we have to find $i_{0} \in\{1, \ldots, N\}$ such that:

$$
t_{i_{0}}^{\sum n_{k} a_{t o k}} \notin\left\{1, x_{i_{0}}^{(1)}, \ldots, x_{i_{0}}^{(r)}\right\} .
$$

As $t$ is non-zero, let us fix $\tau \in \mathbb{C}$ such that $t=\exp (2 i \pi \tau)$. As $t$ is not a root of 1 , $\tau \notin \mathbb{Q}$. As each $x_{i}^{(s)}$ is not zero, we fix $y_{i}^{(s)}$ such that $x_{i}^{(s)}=\exp \left(2 i \pi y_{i}^{(s)}\right)$. Then, an equality $t_{l}^{\sum n_{k} a_{i k}}=x_{i}^{(s)}$ gives:

$$
\frac{\left(\alpha_{i} \mid \alpha_{i}\right)}{2} \sum_{1}^{N} n_{k} a_{i k}=y_{i}^{(s)}+\frac{m}{\tau} \text { for a certain } m,
$$




$$
\sum_{1}^{N} n_{k}\left(\alpha_{i} \mid \alpha_{k}\right)=y_{i}^{(s)}+\frac{m}{\tau}
$$

As the left-hand side belongs to $\mathbf{Z}$, the right-hand side must also, and as there is at most an integer $m$ such that $y_{i}^{(s)}+m / \tau \in \mathbf{Z}$. Let us put $z_{i}^{(s)}=y_{i}^{(s)}+m / \tau$. Suppose that for each $i \in\{1, \ldots, N\}$, there exists $s \in\{0, \ldots, r\}$ such that

$$
\sum_{k=1}^{N} n_{k}\left(\alpha_{i} \mid \alpha_{k}\right)=z_{i}^{(s)}
$$

We have a linear system, with unknowns $\left(n_{1}, \ldots, n_{N}\right)$ and matrix $\left(\left(\alpha_{i} \mid \alpha_{k}\right)\right)$ which is invertible. So, given $\left(z_{1}^{\left(s_{1}\right)}, \ldots, z_{N}^{\left(s_{N}\right)}\right)$, there is at most an integral solution to the system. As we can form only a finite number of $N$-uples $\left(z_{1}^{\left(s_{1}\right)}, \ldots, z_{N}^{\left(s_{N}\right)}\right)$, we see that if $\left(n_{1}, \ldots, n_{N}\right)$ is not in a certain finite set, there is always an index $i_{0}$ such that: $t_{i_{0}}^{\sum n_{k} a_{i_{0} k}} \notin\left\{1, x_{i_{0}}^{(1)}, \ldots, x_{i_{0}}^{(s)}\right\}$. Let $M=\sup \left(\left|n_{1}\right|+\left|n_{2}\right|+\cdots+\left|n_{N}\right|\right)+1$, where $\left(n_{1}, \ldots, n_{N}\right)$ belongs to the excluded finite set, and we get the lemma.

Proposition 4. Let $V$ be a cyclic $U_{t} \mathscr{G}$-module generated by a highest weight vector $v_{+}$, with weight $\lambda=\left(\lambda_{1}, \ldots, \lambda_{N}\right)$.

1) $V$ is spanned by $v_{+}$and the $\rho\left(f_{i_{1}}\right) \cdots \rho\left(f_{i_{p}}\right) v_{+}, i_{1}, \ldots, i_{p} \in\{1, \ldots, N\}$, and such $a$ vector, if non-zero, is a vector of weight $\mu=\left(\mu_{1}, \ldots, \mu_{N}\right)$ with $\mu_{k}=\lambda_{k} \cdot t_{k}^{-\sum_{j} a_{k i j}}$.

2) All the weights of $V$ are of this form.

3) For each weight $\mu$, $\operatorname{dim} V_{\mu}<\infty$ and $\operatorname{dim} V_{\lambda}=1$.

4) $V$ is an indecomposable $U_{t} \mathscr{G}$-module, with a unique maximal proper submodule.

Proof. (Compare Humphreys [4]). Quite analogous to the classical one, using the decomposition $U_{t} \mathscr{G}=U_{t} n_{-} \otimes \mathbb{C}[T] \otimes U_{t} n_{+}$. (For 3), use the same argument as in Lemma 5 to prove that $\rho\left(f_{j_{1}}\right) \cdots \rho\left(f_{j_{r}}\right) v_{+}$and $\rho\left(f_{i_{1}}\right) \cdots \rho\left(f_{i_{p}}\right) v_{+}$have the same weight iff $\forall i, f_{i}$ appears the same number of times in $\left\{f_{j_{2}}, \ldots, f_{j_{r}}\right\}$ and in $\left\{f_{i_{1}}, \ldots, f_{i_{p}}\right\}$.

Proposition 5. If $\rho$ and $\rho^{\prime}$ are irreducible representations with the same highest weight, they are equivalent.

Now, given an irreducible finite dimensional representation, we know that it has highest weight, necessarily unique. In order to determine the possible values of $\lambda=\left(\lambda_{1}, \ldots, \lambda_{N}\right)$, we shall consider, for each $i=1, \ldots, N$, the restriction of the representation to the subalgebra generated by $k_{i}^{ \pm 1}, e_{i}, f_{i}$ (which is isomorphic to $\left.U_{t} \mathrm{~s}(2)\right)$.

\section{Finite Dimensional Representations of $U_{t} \mathrm{sl}(2)$}

We shall call $k^{ \pm 1}, e, f$ the generators.

Theorem 1. 1. If $\lambda \in \mathbf{C}^{*}$ is the highest weight of a finite dimensional representation of $U_{t} \mathrm{~s} 1(2)$, then $\lambda=\omega \cdot t^{m}$, where $\omega \in\{1,-1, i,-i\}, m \in \mathbf{N}$.

2. For each $m \in \mathbf{N}$ and $\omega \in\{1,-1, i,-i\}, \lambda=\omega \cdot t^{m}$ is the highest weight of an irreducible representation of dimension $(m+1)$, and the weights of this representation are exactly: $\omega t^{m}, \omega t^{m-2}, \ldots, \omega t^{-m}$.

3. Every finite dimensional representation of $U_{t} \mathrm{sl}(2)$ is completely reducible. 
Proof. 1.Let $v$ be a vector with highest weight $\lambda$ and put, for $p \in \mathbf{N}, v_{p}=(1 / p !) \rho(f)^{p} \cdot v$. Then:

i) $\rho(f) v_{p}=(p+1) v_{p+1}$

ii) $\rho(k) v_{p}=\lambda t^{-2 p} v_{p}$

and the formula $\left[e, f^{p}\right]=f^{p-1}\left(\left(t^{2 p}-t^{-2 p}\right) /\left(t^{2}-t^{-2}\right)\right) \cdot\left(\left(k^{2} t^{-2(p-1)}-\right.\right.$ $\left.\left.k^{-2} t^{2(p-1)}\right) /\left(t^{2}-t^{-2}\right)\right)$ and the fact that $\rho(e) \cdot v=0$, show that we have:

iii) $\rho(e) v_{p}=\frac{t^{2 p}-t^{-2 p}}{\left(t^{2}-t^{-2}\right)} \cdot \frac{t^{-2(p-1)} \lambda^{2}-t^{2(p-1)} \lambda^{-2}}{\left(t^{2}-t^{-2}\right)} v_{p-1}, p \geqq 1$.

As $V$ is finite dimensional, there is a first integer $m$ such that $v_{m}=0$. Then, as $t$ is not a root of $1, \lambda^{4}=t^{4(m-1)}$, so $\lambda=\omega t^{m-1}, \omega \in\{1,-1, i,-i\}$.

2. Let $V$ be a $\mathbf{C}$-vector space with basis $\left(v_{0}, \ldots, v_{m}\right)$, on which $k, e, f$ act by the same formulas i), ii), iii) with $\lambda=\omega t^{m}$. Then $\rho(k), \rho(e), \rho(f)$ verify the defining relations of $U_{t} \mathrm{sl}(2)$ : so $(\rho, v)$ is a representation of $U_{t} \mathrm{sl}(2)$ and it is irreducible since the $v_{p}$ 's are the only weight vectors possible (up to scalar).

3. We have to check that if $V$ is a finite dimensional $U_{t} \operatorname{sl}(2)$-module and $V^{\prime}$ an invariant subspace of $V$, then there is an invariant subspace $V^{\prime \prime}$ such that $V=V^{\prime} \oplus V$.

a) Case where $V^{\prime}$ is of Codimension 1. By using induction on the dimension of $V^{\prime}$, one classically reduces to the case where $V^{\prime}$ is also irreducible; so, it is a highest weight module. Let us call $\omega \cdot t^{m}$ its highest weight.

Lemma 6. 1. $C=\left(\left(k t-k^{-1} t^{-1}\right)^{2} /\left(t^{2}-t^{-2}\right)^{2}\right)+f e$ is in the center of $U_{t} \mathrm{~s} 1(2)$ and it acts in every finite dimensional irreducible representation, by a non-zero scalar. (Compare Jimbo [3]).

2. For $\omega^{\prime} \in\{1,-1, i,-i\}$, let $C^{\prime}=C-\left(\omega^{\prime} t-\omega^{\prime-1} t^{-1}\right)^{2} /\left(t^{2}-t^{-2}\right)^{2}$. It acts in every finite dimensional irreducible representation by a non-zero scalar if the dimension of the representation is greater than 2 .

Proof. One checks immediately that $C$ and $C^{\prime}$ commute with $e, f, k$. So, they are in the center of $U_{t} \operatorname{sl}(2)$ and act by a scalar in every irreducible representation. This scalar is obtained by evaluating on the highest weight vector $v_{0}$. For $C$, one gets $\left(\left(\omega t^{m+1}-\omega^{-1} t^{-(m+1)} /\left(t^{2}-t^{-2}\right)\right)^{2}\right.$ which is non-zero as $t$ is not a root of 1 .

For $C^{\prime}$, one gets: $\left(\left(\omega^{2} t^{2(m+1)}+\omega^{-2} t^{-2(m+1)}-\omega^{\prime 2} t^{2}-\omega^{\prime-2} t^{-2}\right) /\left(t^{2}-t^{-2}\right)^{2}\right)$.

But $\omega^{2}=\omega^{-2}$ and $\omega^{\prime 2}=\omega^{\prime-2}$.

It is zero if and only if $\omega^{2}\left(t^{2(m+1)}+t^{-2(m+1)}\right)=\omega^{\prime 2}\left(t^{2}+t^{-2}\right)$,

But

$$
\frac{t^{2(m+1)}+t^{-2(m+1)}}{t^{2}+t^{-2}}=\left(\frac{\omega^{\prime}}{\omega}\right)^{2} \in\{1,-1\} \text {. }
$$

$$
\frac{t^{2(m+1)}+t^{-2(m+1)}}{t^{2}+t^{-2}}=1 \Leftrightarrow t^{2}\left(t^{2 m}-1\right)=t^{-2(m+1)}\left(t^{2 m}-1\right)
$$

impossible if $t$ is not a root of $1(m \geqq 1$ as the dimension of the representation is $m+1)$

$$
\frac{t^{2(m+1)}+t^{-2(m+1)}}{t^{2}+t^{-2}}=-1 \Leftrightarrow t^{2}\left(t^{2 m}+1\right)=t^{-2(m+1)}\left(t^{2 m}+1\right)
$$

impossible if $t$ is not a root of 1 . 
Proof of a). Suppose first that $\operatorname{dim} V^{\prime} \geqq 2$.

Consider the representation of $U_{t} \mathrm{sl}(2)$ in $V / V^{\prime}$, which is 1-dimensional: $e_{i}, f_{i}$ act by 0 and $k_{i}$ by a scalar $\omega^{\prime} \in\{1,-1, i,-i\}$. Define $C^{\prime}$ as in the lemma and let it act in $V$ : it takes $V^{\prime}$ into $V^{\prime}$, where it acts by a non-zero scalar according to Lemma 6 , and in fact it takes $V$ into $V^{\prime}$ as it acts by 0 in $V / V^{\prime}$ (by choice of $\omega^{\prime}$ ). So $V_{2}=\operatorname{ker} C^{\prime}$ is 1-dimensional and $V=V^{\prime} \oplus V_{2}$. Furthermore, $V_{2}$ is invariant under $U_{t} \mathrm{sl}(2)$ as $C^{\prime}$ belongs to the center.

Suppose now $\operatorname{dim} V^{\prime}=1$ and $\operatorname{dim} V=2$. The only non-trivial case is the one where $\omega$, the weight of the representation in $V^{\prime}$ is equal to $\omega^{\prime}$, the weight of the representation in $V / V^{\prime}$. So, there exists a basis $\left(v_{1}, v_{2}\right)$ in $V$ in which $\rho(k)$ has matrix $\left(\begin{array}{ll}\omega & \alpha \\ 0 & \omega\end{array}\right), \alpha \in \mathbf{C}$.

Then $\rho(k)\left[\rho(e) v_{1}\right]=t^{2} \omega \rho(e) v_{1}$, so $\rho(e) v_{1}=0$.

Then $\rho(k)\left[\rho(e) v_{2}\right]=t^{2} \rho(e)\left[\omega v_{2}+\alpha v_{1}\right]=t^{2} \omega \rho(e) v_{2}$, so $\rho(e) v_{2}=0$ and $\rho(e)=0$. Similarly, $\rho(f)=0$. $\alpha=0$.

Then the relation $[e, f]=\left(k^{2}-k^{-2}\right) /\left(t^{2}-t^{-2}\right)$ implies $\rho(k)^{2}=\rho\left(k^{-1}\right)^{2}$, so

b) General Case. $V^{\prime}$ of any codimension. Let

$$
\begin{aligned}
\mathscr{V} & =\left\{f \in \mathscr{L}\left(V, V^{\prime}\right) / f_{\mid V^{\prime}} \text { is a scalar operator }\right\}, \\
\mathscr{V}^{\prime} & =\left\{f \in \mathscr{L}\left(V, V^{\prime}\right) / f_{\mid V^{\prime}}=0\right\} .
\end{aligned}
$$

Then $\mathscr{V}^{\prime}$ is a subspace of codimension 1 in $\mathscr{V}$.

One makes $U_{t}$ sl(2) act in $\mathscr{L}\left(V, V^{\prime}\right)$ after identifying $\mathscr{L}\left(V, V^{\prime}\right)$ with $V^{\prime} \otimes V^{*}$ and putting: $\bar{\rho}=(\rho \otimes \tilde{\rho}) \circ \Delta$, where $\tilde{\rho}={ }^{t} \rho \circ S$ is the contragradient representation in $V^{*}$. If one fixes a basis $\left(y_{1}, \ldots, y_{p}\right)$ of $V^{\prime}$, one can write any $\varphi \in \mathscr{L}\left(V, V^{\prime}\right)$ uniquely as $\varphi=\sum y_{i} \otimes x_{i}^{*}$ for some $x_{i}^{*} \in V^{*}$.

One then checks without difficulty that $\mathscr{V}$ and $\mathscr{V}^{\prime}$ are invariant under $\bar{\rho}$. Applying a), there exists an invariant subspace $\mathscr{V}^{\prime \prime}$ such that $\mathscr{V}=\mathscr{V}^{\prime} \oplus \mathscr{V}^{\prime \prime}$. Let $\varphi=\sum y_{i} \otimes x_{i}^{*}$ a non-zero element in $\mathscr{V}^{\prime \prime}:$ it acts in $V^{\prime}$ by a non-zero scalar and $\operatorname{Ker} \varphi=\cap_{i} \operatorname{Ker} x_{i}^{*}$ verifies $V=\operatorname{Ker} \varphi \oplus V^{\prime}$. Furthermore, $\operatorname{Ker} \varphi$ is invariant under $U_{t} \mathscr{G}$ (because $\mathscr{V}^{\prime \prime}$ was) and $\operatorname{Ker} \varphi$ is the sought for space.

Corollary. If $\lambda=\left(\lambda_{1}, \ldots, \lambda_{N}\right)$ is the highest weight of a finite dimensional irreducible representation of $U_{t} \mathscr{G}$, then, necessarily, $\lambda_{k}$ is of the form $\lambda_{k}=\omega_{k} t_{k}^{m_{k}} . \omega_{k} \in\{1,-1$, $i,-i\}, m_{k} \in \mathbf{N}$.

\section{Finite Dimensional Representations of $U_{t} \mathscr{G}$}

1. Any 1-dimensional representation is irreducible, with highest weight $\omega=$ $\left(\omega_{1}, \ldots, \omega_{N}\right) \in\{1,-1, i,-i\}^{N}$. Let us denote it by $\left(\rho_{\omega}, \mathbb{C}_{\omega}\right)$. If $(\rho, V)$ is any finite dimensional irreducible representation, with highest weight $\lambda$, then $\left(\rho \otimes \rho_{\omega}\right) \circ \Delta$ gives an irreducible representation in $V \otimes \mathbb{C}_{\omega}$, with highest weight $\omega . \lambda=$ $\left(\omega_{1} \lambda_{1}, \ldots, \omega_{N} \lambda_{N}\right)$.

2. Let $\tilde{\lambda}$ a dominant weight of $\mathscr{G}$ (with the basis of roots $\left(\alpha_{i}\right)$ ). One can associate to it a character of $T$, noted $t^{\lambda}$, by: $t^{\lambda}\left(k_{i}\right)=t_{i}^{\chi(H)}$, where $\left(H_{1}, \ldots, H_{N}\right)$ is the coroot system associated with $\left(\alpha_{1}, \ldots, \alpha_{N}\right)$. 
The corollary shows that to each highest weight $\lambda$, one can associate a 1-dimensional representation $\left(\rho_{\omega}, \mathbb{C}_{\omega}\right)$ and a dominant weight $\tilde{\lambda}$ defined by $\tilde{\lambda}\left(H_{i}\right)=\left\langle\tilde{\lambda}, \alpha_{i}\right\rangle=m_{i} \in \mathbb{N}$.

This is the first point of the following theorem:

\section{Theorem 2.}

1. If $(\rho, V)$ is a finite dimensional irreducible representation with highest weight $\lambda$, then $\lambda=\omega . t^{\lambda}$, where $\omega \in\{1,-1, i,-i\}^{N}$ and $\tilde{\lambda}$ is a dominant weight of $\mathscr{G}$.

2. Any character of $T$ of this form is the highest weight of a finite dimensional irreducible representation.

3. Any finite dimensional representation of $U_{t} \mathscr{G}$ is completely reducible.

Proof.

2. According to the remarks in 1., we only have to consider the case where $\lambda=t^{\tilde{\hat{\lambda}}}$. But, for each $\lambda \in\left(\mathbf{C}^{*}\right)^{N}$, one can construct the universal standard cyclic module with highest weight $\lambda$, call it $Z(\lambda)$, by an induced module construction: consider the 1-dimensional space $D_{\lambda}$, with basis $v_{+}$, on which $U_{t} b_{+}$acts as follows:

$$
\begin{aligned}
& e_{i} \cdot v_{+}=0 \forall i \\
& k_{i} \cdot v_{+}=\lambda_{i} v_{+} \forall i .
\end{aligned}
$$

Put $Z(\lambda)=U_{t} \mathscr{G} \bigotimes_{U_{t} b_{+}} D_{\lambda}$ : it is a left $U_{t} \mathscr{G}$-module in which $1 \otimes v_{+}$is not zero because $U_{t} \mathscr{G}$ is a free right $U_{t} b_{+}$-module, and $1 \otimes v_{+}$generates $Z(\lambda)$. Taking the quotient by the maximal proper submodule (see Prop. 4), we get an irreducible module with highest weight $\lambda: V(\lambda)$. The fact that, when $\tilde{\bar{\lambda}}$ is dominant, $V\left(t^{\tilde{\hat{\lambda}}}\right)$ is finite dimensional will follow from:

Proposition 6. Let $V\left(t^{\tilde{\hat{\lambda}}}\right)$ the irreducible module as above, where the dominant weight $\tilde{\lambda}$ is defined by the positive integers $m_{i}=\tilde{\lambda}\left(H_{i}\right)$. Then:

1. $f_{i}^{m_{i}+1} \cdot v_{+}=0 \quad \forall i=1, \ldots, N$.

2. For each $1 \leqq i \leqq N, V\left(t^{\tilde{\lambda}}\right)$ contains a non-zero finite dimensional $L_{i}$-module $\left(L_{i}\right.$ is the subalgebra generated by $\left.e_{i}, f_{i}, k_{i}^{ \pm 1}\right)$.

3. $V\left(t^{\tilde{\hat{\lambda}}}\right)$ is the sum of the finite dimensional $L_{i}$-submodules.

4. The Weyl group $W$ acts on the set $P$ of weights. Each weight subspace $V_{\mu}$ is finite dimensional and $\operatorname{dim} V_{\sigma \mu}=\operatorname{dim} V_{\mu} \forall \sigma \in W$.

5. The set of weights $P$ is finite.

Then, $V\left(t^{\tilde{\hat{\lambda}}}\right)$ being irreducible, it equals the sum of its weight subspaces and 4. and 5. show that it is finite dimensional.

Proof of proposition (Compare Humphreys [4]).

1. Let $w=f_{i}^{m_{l}+1} \cdot v_{+}$and let us show that, if $w \neq 0$, it is a highest weight vector, with highest weight different from $t^{\tilde{i}}$ (such a vector cannot exist as $V\left(t^{\tilde{i}}\right)$ is irreducible). First, $k_{j} \cdot w=t_{j}^{-a_{\jmath 2}\left(m_{1}+1\right)} f_{i}^{m_{2}+1} k_{j} v_{+}=t_{j}^{-a_{j i}\left(m_{1}+1\right)} t_{j}^{\lambda\left(H_{j}\right)} w$. So, if $w \neq 0$, it is a weight vector with weight $t^{\tilde{\lambda}-\left(m_{t}+1\right) \alpha_{i}} \neq t^{\lambda}$. Then, as for $i \neq j, e_{j}$ and $f_{i}$ commute, $e_{j} \cdot w=0$. For $i=j$, the relation

$$
\left[e_{i}, f_{i}^{m_{i}+1}\right]=f_{i}^{m_{i}} \cdot \frac{t_{i}^{2\left(m_{i}+1\right)}-t_{i}^{-2\left(m_{1}+1\right)}}{t^{2}-t^{-2}} \cdot \frac{k_{i}^{2} t_{i}^{-2 m_{i}}-k_{i}^{-2} t_{i}^{2 m_{\imath}}}{t^{2}-t^{-2}}
$$


and the fact that $k_{i} \cdot v_{+}=t_{i}^{m_{1}} v_{+}$shows that $e_{i} \cdot w=0$. So $w$ would be a highest weight vector.

2. For $1 \leqq i \leqq N$, consider the subvectorspace spanned by $v_{+}, f_{i} \cdot v_{+}, \ldots, f_{i}^{m_{1}+1}$. $v_{+}$. Commutation rules between $e_{i}, f_{i}$ and $k_{i}$ show that it is invariant under $L_{i}$.

3. Let $V^{\prime}$ the sum of the finite dimensional $L_{i}$-submodules. According to 2 ), $V^{\prime} \neq\{0\}$. To check that $V^{\prime}=V\left(t^{\tau}\right)$, it is enough to see that it is invariant under all $e_{j}, f_{j}, k_{j}$.

Remark. $1-a_{i j} \in\{1, \ldots, 4\}$. If $1-a_{i j}=1$, then $e_{i} e_{j}=e_{j} e_{i}$. For $1-a_{i j} \geqq 2$, put $e_{i, j}=$ $e_{i} e_{j}-t_{i}^{2 a_{i j}} e_{j} e_{i}$. Then, if $1-a_{i j}=2$, one defining relation gives $e_{i} e_{i, j}-t_{i}^{4+2 a{ }^{j}} e_{i, j} e_{i}=$ 0 . If $1-a_{i j}=3$, put $e_{i, i, j}=e_{i} e_{i, j}-t_{i}^{4+2 a j} e_{i, j} e_{i}$, and we have $e_{i} e_{i, i, j}=t_{i}^{8+2 a^{1 j}} e_{i, i, j} e_{i}$. For $1-a_{i j}=4$, put $e_{i, i, i, j}=e_{i} e_{i, i, j}-t_{i}^{8+2 a{ }^{\prime}} e_{i, i, j} e_{i}$ and then: $e_{i} e_{i, i, i, j}=t^{12+2 a{ }^{j}} e_{i, i, i, j} e_{i}$. Same remark with the $f_{i}$, s. Now, the invariance of $V^{\prime}$ will result from the following fact: if $W$ is an invariant finite dimensional $L_{i}$-submodule, then the vector space spanned by $e_{j} W, f_{i} W, k_{J} W, \quad e_{i, j} W, f_{i, j} W, \ldots, e_{i, i, i, j} W$ and $f_{i, i, i, j} W$ (where $j \in\{1, \ldots, N\} \backslash\{i\})$ is finite dimensional and invariant under $L_{i}$ according to the remark. So, $U_{t} \mathscr{G}(W) \subset V^{\prime}$.

4. The finite dimensionality of each $V$ is proved as in Proposition 4. Let $\mu=t^{\tilde{\mu}} \in P$ and $\sigma_{i} \in W$ associated with the simple root $\alpha_{i}$. Let us show that $\sigma_{i}\left(t^{\tilde{\mu}}\right)$, defined as $t^{\sigma_{i}(\tilde{\mu})}$, belongs to $P$. But the subspace $\bigoplus_{k \in \mathbf{Z}} V_{t^{\tilde{\mu}+k \alpha_{i}}}$ is invariant under $L_{i}$; let us fix $v_{\mu} \in V_{\mu} \backslash\{0\}$. According to 3 ), there is a non-trivial finite dimensional subspace $V^{\prime \prime}$ of $\oplus V_{t \tilde{\mu}+k x_{i}}$, invariant under $L_{i}$ and containing $v_{\mu}$. According to the complete reducibility theorem for $U_{t_{2}} \mathrm{sl}(2), V^{\prime \prime}$ is a direct sum of irreducible $L_{i}$-modules. As $\mu=t^{\tilde{\mu}}$ is a weight for the representation in $V^{\prime \prime}, \mu_{i}=t_{i}^{\tilde{\mu}\left(H_{\imath}\right)}$ appears as a weight of one of the irreducible summands. According to Theorem $1, t_{i}^{-\tilde{\mu}\left(H_{i}\right)}$ is also a weight for this irreducible $L_{i}$-module. But, as the possible weights are restrictions of those of $V^{\prime \prime}$, there is $k$ in $\mathbf{Z}$ such that:

But

$$
t_{i}^{-\tilde{\mu}\left(H_{\imath}\right)}=t_{i}^{\tilde{\mu}\left(H_{\imath}\right)+k \alpha_{\imath}\left(H_{\imath}\right)} \text {, that is } 2 \tilde{\mu}\left(H_{i}\right)=-k \alpha_{i}\left(H_{i}\right) .
$$

So, $t^{\sigma_{1}(\tilde{\mu})} \in P$.

$$
\sigma_{i}(\tilde{\mu})=\tilde{\mu}-\frac{2 \tilde{\mu}\left(H_{i}\right)}{\left(\alpha_{i}, \alpha_{i}\right)} \alpha_{i}=\tilde{\mu}+k \alpha_{i} .
$$

5. Using 4 , the proof is exactly the same as the classical one.

Proof of Point 3) in Theorem 2. (Complete reducibility) We shall use a result due to Professor A. Borel, which he has obtained as a generalisation of an argument allowing him to prove the complete reducibility theorem for complex semi-simple Lie algebras without using the Casimir operator.

His result is the following:

Theorem (A Borel): Let $A$ be an algebra, $M$ an additive category of $A$-modules and $\mathscr{S}$ the set of classes of simple A-modules in M. Assume:

1. $M$ is closed under the formation of subquotients. Every element of $M$ has a finite Jordan-Holder series.

2. There is an involutive functor $V \rightarrow V^{*}$ on $M$, reversing the arrows, preserving $\mathscr{S}$, direct sums and short exact sequences.

3. There is a partial order $\leqq$ in $\mathscr{S}$ such that $V \leqq W \Rightarrow V^{*} \leqq W^{*}$. (In the sequel, write $<$ for $\supseteqq$. 
4. Let $0 \rightarrow U \rightarrow V \rightarrow W \rightarrow 0$ be a short exact sequence in $M$, with $U$ and $W$ in $\mathscr{S}$. If $V$ is indecomposable, then $U<W$.

Then under those conditions, every element of $M$ is a direct sum of elements in $\mathscr{S}$.

In fact, Borel's proof remains true if one replaces (2) by the little more general hypothesis:

$\left(2^{\prime}\right)$ There are two functors $F_{1}$ and $F_{2}$ on $M$, reversing arrows, preserving $\mathscr{S}$, direct sums and exact sequences, and which are inverse one of the other. Then, (3) must be true for $F_{1}$ and $F_{2}$.

It is under this form that we shall apply the result to $A=U_{t} \mathscr{G}$ and to the additive category of finite dimensional $U_{t} \mathscr{G}$-modules.

Let us check that the four conditions are satisfied:

1. is clear.

2. Let $F_{1}$ the functor contragredient representation $(\rho, V) \rightarrow\left(\rho_{1}, V^{*}\right)$, where $\rho_{1}={ }^{t} \rho \circ S\left(S\right.$ is the antipode), and $F_{2}$ the functor skew contragredient representation: $(\rho, V) \rightarrow\left(\rho_{2}, V^{*}\right)$, where $\rho_{2}={ }^{t} \rho \circ S^{\prime}\left(S^{\prime}\right.$ is the skew antipode: $S^{\prime}: U_{t} \mathscr{G} \rightarrow U_{t} \mathscr{G}$ is linear, antimultiplicative and inverse of $S$ ).

3. An element in $S$ is characterised by its highest weight $\omega . t^{\varkappa}$, where $\omega \in\{1,-1$, $i,-i\}$ and $\tilde{\lambda}$ is a dominant weight in $\mathscr{G}$.

Let $\preccurlyeq$ the usual partial order on the weights in $\mathscr{G}$. Define the order on $\mathscr{S}$ by:

$$
\omega \cdot t^{\tilde{\lambda}} \leqq \omega^{\prime} \cdot t^{\tilde{\lambda}^{\prime}} \Leftrightarrow \omega=\omega^{\prime} \text { and } \tilde{\lambda} \preccurlyeq \tilde{\lambda}^{\prime} \text {. }
$$

As $S\left(k_{i}\right)=S^{\prime}\left(k_{i}\right)=k_{i}^{-1}, \omega \cdot t^{\tilde{\mu}}$ is a weight in $(\rho, V) \Leftrightarrow \omega^{-1} \cdot t^{-\tilde{\mu}}$ is a weight in $\left(\rho_{1}, V^{*}\right)$ (or $\left(\rho_{i}, V^{*}\right)$ ).

So, to prove $V \leqq W \Rightarrow V^{*} \leqq W^{*}$, we are back to the classical case: if $w_{0}$ is the longest element of the Weyl group $W$, then $i=-w_{0}$ defines an involution in $\mathfrak{h}^{*}$ preserving the weight lattice and the order on it. On the set of characters of $T$ of the form $\omega \cdot t^{\tilde{\mu}}$, where $\omega \in\{1,-1, i,-i\}^{N}$ and $\tilde{\mu}$ is a weight of $\mathscr{G}$, one has an involution $I$ given by: $I\left(\omega . t^{\tilde{\mu}}\right)=\omega^{-1} \cdot t^{(\tilde{\mu})}$, which preserves the order. Now, it is easy to see that if $V$ is an irreducible $U_{t} \mathscr{G}$-module with highest weight $\omega . t^{\tilde{\lambda}}$, then $F_{1}(V)$ and $F_{2}(V)$ are irreducible with highest weight $\omega^{-1} \cdot t^{t(\tilde{\lambda})}$.

4. We shall follow Borel's proof for the classical case.

Let $O \rightarrow V\left(\omega . t^{\tilde{\lambda}}\right) \rightarrow V \rightarrow V\left(\omega^{\prime} \cdot t^{\tilde{\mu}}\right) \rightarrow 0$ be a short exact sequence with $V$ indecomposable. Then $V$ is cyclic with respect to any vector $v$ not contained in $V\left(\omega . t^{\tilde{\lambda}}\right)$. Put $\lambda=\omega \cdot t^{\tilde{\lambda}}, \mu=\omega \cdot t^{\tilde{\mu}}$.

Let us show that $\lambda \neq \mu$. If not, $V_{\lambda}$ is 2-dimensional and there is no weight $v>\lambda$ in $V$. So $V_{\lambda}$ is killed by $U_{t} n_{+}$. So, any $v \in V_{\lambda} \backslash\{0\}$ is a highest weight vector and generates an irreducible submodule whose intersection with $V_{\lambda}$ is 1-dimensional. As $\operatorname{dim} V(\lambda)_{\lambda}=1$, taking $v \in V_{\lambda} \backslash V(\lambda)_{\lambda}$, we see that the cyclic module generated by $v$ should be $V$. Contradiction.

So, $\lambda \neq \mu$. We shall prove that there is in $V \backslash V(\lambda)$ a vector with weight $\mu$ killed by $U_{t} n_{+}$. As such a vector must generate $V$, it will follow that $\lambda<\mu$. Let us note that the space $V^{U_{t^{n}}}$ of vectors killed by $U_{t} n_{+}$is 2 -dimensional: $\operatorname{dim} V^{U^{n_{+}}} \leqq 2$ because $\operatorname{dim} V(\lambda)^{U_{t} n_{+}}=\operatorname{dim} V(\mu)^{U_{t} n_{+}}=1$, and $\operatorname{dim} V^{U^{n_{+}}} \geqq 1$ because $V(\lambda)^{U_{t} n_{+}} \subset$ $V^{U^{n^{n}}+}$. One can suppose that $\mu$ is not $<\lambda$, because, if $\mu<\lambda$, taking the dual exact sequence $0 \rightarrow V(\mu)^{*} \rightarrow V^{*} \rightarrow V(\lambda)^{*} \rightarrow 0$, one has $I(\mu)<I(\lambda)$ and in particular $I(\lambda)$ is not $<I(\mu)$. So one gets $\operatorname{dim}\left(V^{*}\right)^{U_{t} n_{+}}=2$, but as $\left(V^{*}\right)^{U_{t} n_{+}}$is the dual of $V / U_{t} n_{1} . V$, it has the same dimensions as $V^{U_{t} n_{+}}$. 
So suppose that $\mu$ is not $<\lambda$. Then $V$ cannot have weights $v<\mu$ because such a weight would be necessarily a weight of $V(\lambda)$ and we should have $\mu<v \leqq \lambda$. Now, there is $x \in V$ whose image in $V(\mu)$ generates $V(\mu)_{\mu}$. So, for $i=1, \ldots, N, \rho\left(k_{i}\right) x-$ $\mu_{i} x \in V(\lambda)$ and $\rho\left(e_{i}\right) x \in V(\lambda)$. Put $y_{i}=\rho\left(k_{i}\right) x-\mu_{i} x \in V(\lambda)$, and note that $\left(\rho\left(k_{j}\right)-\right.$ $\left.\mu_{j}\right) y_{i}=\left(\rho\left(k_{i}\right)-\mu_{i}\right) y_{j}$. As $\mu$ is not $<\lambda$ it cannot be a weight in $V(\lambda)$; so, there is $i \in\{1, \ldots, N\}$ such that: $\rho\left(k_{i}\right)-\left.\mu_{i}\right|_{V(\lambda)}$ is invertible. Put $z=\left(\rho\left(k_{i}\right)-\mu_{i}\right)^{-1}\left(y_{i}\right) \in V(\lambda)$. Then $\left(\rho\left(k_{j}\right)-\mu_{j}\right) z=y_{j}=\left(\rho\left(k_{j}\right)-\mu_{j}\right) x$. So $x^{\prime}=x-z$ is such that: $\forall i, \quad\left(\rho\left(k_{i}\right)-\right.$ $\left.\mu_{i}\right) x^{\prime}=0, \rho\left(e_{i}\right) x^{\prime} \in V(\lambda)$ and has the same image as $x$ in $V(\lambda)$. Let us show that in fact $\rho\left(e_{i}\right) x^{\prime}=0 \forall i$.

If not, let $i$ such that $\rho\left(e_{i}\right) x^{\prime} \in V(\lambda) \backslash\{0\}$. Then $\forall j \in\{1, \ldots, N\}$,

$$
\rho\left(k_{j}\right) \rho\left(e_{i}\right) x^{\prime}=t_{j}^{a_{j i}} \rho\left(e_{i}\right) \rho\left(k_{j}\right) x^{\prime}=t_{j}^{a_{i j}} \mu_{j} \rho\left(e_{i}\right) x^{\prime}=\omega_{j}^{\prime} t_{j}^{\left(\check{\mu}+\alpha_{i}\right)\left(H_{j}\right)} \rho\left(e_{i}\right) x^{\prime} .
$$

So $\rho\left(e_{i}\right) x^{\prime}$ should be a vector with weight $\omega^{\prime} \cdot t^{\tilde{\mu}+\alpha_{i}}>\omega^{\prime} \cdot t^{\tilde{\mu}}=\mu$. Impossible. So, $x^{\prime}$ is the sought for vector and we have also proved that $\operatorname{dim} V^{U_{r} n_{+}}=2$.

The only remaining case is the one where $\mu<\lambda$, with $\operatorname{dim} V^{U^{n}+}=2$. As $\operatorname{dim} V(\lambda)^{U_{t^{n}}+}=1$, there is an $x \in V^{U_{t^{n}}} \backslash V(\lambda)^{U_{t} n_{+}}$. Its image in $V(\mu)$ is not zero and is killed by $U_{t} n_{+}$. So each of its components $\bar{x}_{v}$ in the decomposition $V(\mu)=\oplus V(\mu)_{v}$ is a highest weight vector if $\bar{x}_{v} \neq 0$. So, as $V(\mu)$ is irreducible, only $\bar{x}_{\mu} \neq 0$. So the $\mu$-component $x_{\mu}$ of $x$ is not zero and, as it is also killed by $U_{t} n_{+}$, it is the sought for vector.

The theorem is now completely proved.

These results have been announced in [7].

Acknowledgements. I would like to thank Professor A. Borel for communicating to me his results on the complete reducibility of finite dimensional representations of semi-simple Lie algebras. It is also a pleasure to thank Professor P. Cartier and Professor A Connes for constant encouragement and interest in my work.

\section{References}

1. Borel, A.: On the complete reducibility of linear complex semi-simple Lie algebras. Private communication

2. Drinfeld, V. G.: Hopf algebras and the quantum Yang-Baxter equation. Sov. Math. Dokl. 32, 254-258 (1985)

3. Drinfeld, V. G.: Quantum groups. Proc. I.C.M. Berkeley, 1986

4. Humphereys, J. E.: Introduction to Lie algebras and Representation Theory. Graduate Texts in Mathematics Vol. 9. Berlin, Heidelberg, New York: Springer

5. Jimbo, M.: A $q$-difference analog of $U(\mathscr{G})$ and the Yang-Baxter equation. Lett. Math. Phys. 10, 63-69 (1985)

6. Jimbo, M.: A $q$-analog of $U(g l(N+1))$, Hecke algebras and the Yang-Baxter equation. Lett. Math. Phys. 11, 247-252 (1986)

7. Rosso, M.: Représentation irréductibles de dimension finie du $q$-analogue de l' algèbre enveloppante d' une algebre de Lie simple. C.R.A.S. Paris. t. 305. Série I. 587-590 (1987)

Communicated by A. Connes

Received February 1, 1988 
